\title{
Arthroscopic treatment of synovial chondromatosis of hip joint
}

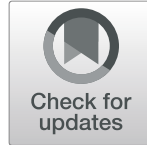

\author{
Yufeng $\mathrm{Liu}^{1+}$, $\mathrm{Ji} \mathrm{Li}^{1+}$, Ning $\mathrm{Ma}^{2}$, Mingyang An${ }^{1}$, Feng Gao ${ }^{3}$, Bo Hu${ }^{4}$, Boqing Zhang ${ }^{1}$, Zhigang Wang ${ }^{1}$, Yujie Liu ${ }^{\text {* }}$ and \\ Chunbao Li $i^{1 *}$
}

\begin{abstract}
Purpose: This retrospective study summarized the clinical, radiographic, and arthroscopic manifestation of synovial chondromatosis (SC) of the hip, along with the post-operative effect to discuss the curative effect of arthroscopic management of hip SC.

Methods: Twenty-one patients who underwent arthroscopic surgery from the same surgeon for hip SC were followed up for an average of 45 months. T-shaped capsulotomy was routinely performed in each case. Visual analog scale, range of motion, modified Harris Hip Score, and International Hip Outcome Tool score were collected preoperatively and at the time of the latest follow-up. All patients' demographics, radiographs, and arthroscopic images were collected to summarize and conclude the similarities and differences of their manifestation.

Results: Large wedged clumps of loose bodies demonstrated distinguishable radiographic, arthroscopic appearance and demanded a different surgical strategy. Postoperative scores were all significantly improved. One case of residual pain and two cases of residual loose bodies with no symptom related were reported at the final follow-up. All but one patient were satisfied with the outcome.

Conclusion: Arthroscopy treatment of hip SC with T-shaped capsulotomy has demonstrated a good result in terms of clinical outcome score, recurrence rate, and complication rate. On the basis of this study, we concluded the clinical performance of large wedged clumps of loose bodies of hip SC.
\end{abstract}

Keywords: Hip arthroscopy, Synovial chondromatosis, Loose body removal, Hip joint

\section{Introduction}

Synovial chondromatosis (SC) is a rare disease with cartilaginous metaplasia which occurs within the synovium of the joint $[1,2]$. It commonly results in the growth of cartilaginous nodules in the synovium, many of which may detach and become loose bodies in the joint and subsequently cause damage to the articular surface and cause osteoarthritis [3]. In the majority of cases, the condition is monoarticular, with hip as the second most frequently affected joint commonly seen on clinical

\footnotetext{
*Correspondence: 13701356381@163.com; lichunbao301@163.com

${ }^{\dagger}$ Yufeng Liu and Ji Li contributed equally to this work.

1 Department of Sports Medicine, Chinese PLA General Hospital, Beijing 100853, China

Full list of author information is available at the end of the article
}

practice [4, 5]. Milgram [3] had defined it as a condition with greater than four osseocartilaginous loose bodies associated with synovial metaplasia.

Early diagnosis of hip SC often means a better prognosis. However, due to the nonspecific and insidious symptom of the disease and regularly false-negative imaging in early stages, the period to confirm definitive diagnosis often exceeds more than 2 years. Complaints usually include nonspecific pain as the main cause for medical consultation, motion limitation (51 to 100\%), limp (9.9 to $90 \%$ ), and locking episodes (19 to 63\%) [6, 7], all of which are commonly seen in various hip conditions. Plain radiograph was considered first-line imaging, due to its easy practicability, sensitivity for the most common

(c) The Author(s). 2020 Open Access This article is licensed under a Creative Commons Attribution 4.0 International License, which permits use, sharing, adaptation, distribution and reproduction in any medium or format, as long as you give appropriate credit to the original author(s) and the source, provide a link to the Creative Commons licence, and indicate if changes were made. The images or other third party material in this article are included in the article's Creative Commons. licence, unless indicated otherwise in a credit line to the material. If material is not included in the article's Creative Commons licence and your intended use is not permitted by statutory regulation or exceeds the permitted use, you will need to obtain permission directly from the copyright holder. To view a copy of this licence, visit http://creativecommons.org/licenses/by/4.0/ The Creative Commons Public Domain Dedication waiver (http://creativecommons.org/publicdomain/zero/1.0/) applies to the data made available in this article, unless otherwise stated in a credit line to the data. 
hip diseases, and common availability, and showed a mean sensitivity of 28 to $83 \%$ [6]. CT and MRI could help confirm the diagnosis with a higher accuracy but more costs. Delaying of treatment could cause complications such as joint deterioration and secondary osteoarthritis (OA), finally leading to arthroplasty or handicap [8]. Conservative treatment for hip SC has shown little effect. A broad consensus about the necessity for operative removal of intra-articular loose bodies has already been reached. As a relatively mature technique, open arthrotomy has shown relatively good results [6, 9-11], but causes bigger trauma and longer time to recover. Hip arthroscopy could be used as an approach of management, but also a tool for definitive diagnosis. Arthroscopic management for hip SC appears safer with an approximate $1 \%$ minor complication rate [12] and a $7.1 \%$ recurrence rate [13]. In addition to that, minimally invasive surgery has been proven to shorten the inhospital stay and postoperative rehabilitation with possible fully weight bearing. However, the complexity of hip's anatomy structure and profound soft tissue around it makes hip arthroscopy a technically demanding procedure. Considering the prognosis of hip SC largely depends on the complete removal of scattering loose bodies all over the joint, it requires extremely high standard for physician's surgical capability.

The aim of this study was to report our own experience of arthroscopic management of hip SC in 21 patients in a retrospective way. T-shaped capsulotomy and synovectomy was performed in all cases. Retrospective analysis of all patients' demographics, radiographs, and arthroscopic images was performed. Reviewing this gave us a clearer look at the typical risk factors, manifestation, and therapeutic effect of this manner.

\section{Materials and methods Patients}

From March 2013 to January 2019, 385 patients had undergone hip arthroscopic surgery and 35 were diagnosed with hip SC according to radiographic, arthroscopic, and pathological manifestation. All 35 patients consented to participate in the study. Demographic data, history, physical examination findings, imaging results, and intraoperative findings were provided. All data were collected prospectively in a computer database following approval by the research ethics board. All operations were performed by the same surgeon (the 2nd corresponding author). Exclusion criteria include the following: (1) history of surgery of the affected hip, (2) grade IV arthritis (Kellgren-Lawrence classification system) under plain radiograph, (3) combined dysfunction of other joint, (4) history of joint infection, (5) history of autoimmune disease, (6) history of chronic consumptions such as tumor and tuberculosis, and (7) less than 2 years of follow-up.

\section{Clinical and radiographic evaluation}

The study was conducted through retrospective examinations of all the patients. We inquired different symptoms from every patient, including pain, locking episode, motion limitation, and limping. FADDIR test and FABER test were conducted in addition to normal physical examination. Visual analog scale (VAS), range of motion (ROM), modified Harris Hip Scores (mHHS), and International Hip Outcome Tool (iHOT12) were measured and inquired before surgery and at final follow-up.

Plain radiographs of anteroposterior views of pelvic with the affected hip joint were taken. KellgrenLawrence classification system was used to assess the grade of OA of hip joint. Radiographic evidence for diagnosis of SC was mainly detection of loose bodies. Medial joint space widening could also be an indicator for reasonable speculation. Other issues which required attention include FAI, osteophytes, subchondral cysts or sclerosis, or bone erosion. In consideration of nonossified loose bodies getting in the way of a confirmative diagnosis, MRI was also performed on all patients preoperatively. Labral tears [4] were detected in MRI, which was also a condition need to be dealt with. Extra attention was paid to the size and location of loose bodies, thickening synovium, and presence of joint effusion for surgery planning.

\section{Surgical technique}

After general anesthesia, the patient was positioned supine in the traction bed with marker made on the skin. The area of perineum was carefully protected. Standard skin preparation and draping was finished. Traction of ipsilateral lower limbs was performed to confirm at least $8-10 \mathrm{~mm}$ of joint-space access, with the hip abducted $10^{\circ}$ to $15^{\circ}$ and internal rotated. An anterolateral approach (ALA) was firstly taken under fluoroscopic control approximately $1 \mathrm{~cm}$ superior and anterior to the anterior edge of the greater trochanter, and then an anteromedial approach (AMA) was established under arthroscopic surveillance. Capsulotomy was performed between two portals. The central compartment was investigated first. Standard surveillance of the acetabulum, labrum, cartilage of femoral head and acetabular fossa, and round ligament was performed. Loose bodies and hyperplasia and hyperemia of synovium were addressed emphatically. Smaller loose bodies were rinsed out with cannula and larger ones were clamped out. Curette, curved scraper, and slide winder blade were used to wipe out the synovial tissue. Cartilage lesions were evaluated according to Outerbridge classification, and then a chondroplasty or microfracture was performed accordingly. Damaged and degraded round ligament was trimmed. Overcoverage of acetabulum (Pincer-type dysplasia) has 
restored normal anatomy by abrasion of hyperplastic bone. Before management of labral pathology, distal anterolateral approach (DALA) was created to facilitate suture and anchor placement to ensure safety. T-shaped capsulotomy was routinely performed along the fiber direction of the iliofemoral ligament to connect the three approaches created. Labrectomy, trimming, or stitches with suture anchor were performed according to the quality and level of damage of the labrum. Peripheral compartment was accessed after central compartment was addressed. Traction was relaxed, and the hip joint was bended at the range of $35 \sim 45^{\circ}$ to restore relaxation state of the articular capsule. Extra attention was paid to explore the anteromedial and posterolateral recess to avoid residue loose bodies and synovium. Cam-type FAI was addressed with abrasion of osteophytosis. Dynamic inspection under arthroscopic surveillance was implemented to ensure no impingement between the acetabular bone and femoral neck during internal and external rotation. Synovectomy was performed thoroughly. Capsule was closed. The number of loose bodies was counted after the operation. Big clumps of loose bodies formed by several small ones were counted as one.

\section{Rehabilitation}

Touchdown weight bearing with crutches was allowed at the 1st day after operation. Passive range of motion was restricted up to $90^{\circ}$. In the first 4 weeks, weight bearing as tolerated with crutches was allowed until full weight bearing was reached. Active range of motion could be gradually increased up to $90^{\circ}$ without external rotation or extension from resting position. Isometric contraction of muscles of the lower extremities was encouraged. At fifth to twelfth week, full weight bearing and normal range of motion were achieved. Daily activity could be restored with or without crutches as needed. Low impact sports as tolerated were encouraged. High-impact sports were not recommended until 6 months.

\section{Statistical analysis}

The SPSS 17.0 (SPSS Inc., Chicago, Illinois) was used to analyze all the data collected. Normal distribution of every data was checked before other analyses. The value of every affected factor, the VAS, ROM, mHHS, and iHOT12 were calculated and analyzed to tell the significance using paired $\mathrm{t}$ tests with $P<0.05$ considered statistically significant.

\section{Results}

\section{Clinical presentation and postoperative outcome}

Twenty-one patients were included into the final group (Fig. 1) with an average follow-up of 45 months (range, 24-69 months). All 21 cases complained symptoms of ipsilateral hip pain, 20 of limited mobility, along with

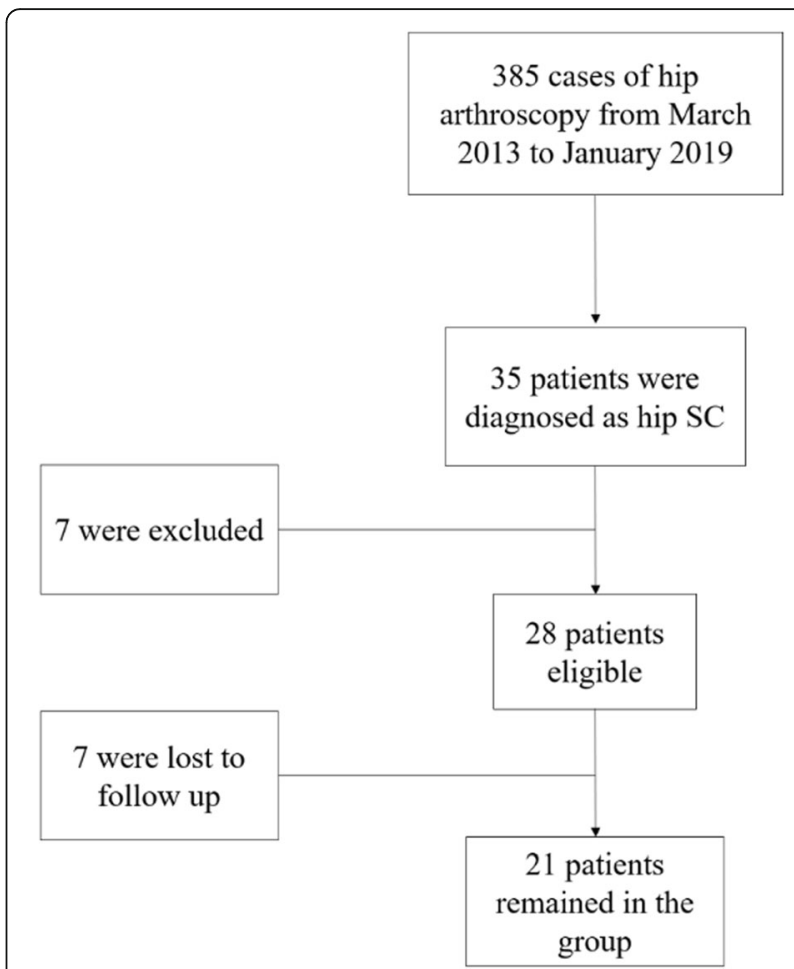

Fig. 1 Flowchart of the enrollment of patients. Twenty-one patients included into the final group

seven cases of locking episodes. Most commonly used terms to describe pain was swelling and dull, some of which accompanied with radiating pain of the ipsilateral thigh and knee. In the 21 patients, 20 (95.2\%) were satisfied with the postoperative outcome. No complaining of pain was presented at the final follow-up except for 1 case. Range of motion was restored back to normal in 20 patients. Locking was gone in all patients. Limping was spotted in 19 out of 21 cases preoperatively, and all gained normal gait at the final follow-up except for one. Atrophy of gluteus medius muscle came up in 17 cases before operation, and only three remained at the final follow-up. FADDIR and FABER tested positive in 14 and 13 cases and were reduced to three and four cases respectively. VAS, ROM, mHHS, and iHOT12 score all improved significantly after the procedure $(P<0.001)$ (Table 1). There was one complication of continuous pain occurred. Ipsilateral hip pain still existed with limited mobility and limping. Atrophy of the gluteus medius muscle of the affected hip still existed after surgery, and there was little improvement of VAS, ROM, mHHS, and iHOT12 score. We contribute this to the late phase development and severe damage of cartilage (Outerbridge stage IV). Progression of arthritis was shown. This patient eventually ended up with total hip replacement. Two cases of residual loose bodies were identified on postoperative plain radiograph without associated 
Table 1 Comparison of clinical outcomes and range of motion

\begin{tabular}{llll}
\hline & Pre-op & Post-op & $P$ value \\
\hline VAS score & $5.90 \pm 0.89$ & $2.24 \pm 1.14$ & $0.0000^{* * *}$ \\
ROM & $153.57 \pm 26.41$ & $245.33 \pm 36.17$ & $0.0000^{* * *}$ \\
mHHS & $36.43 \pm 7.05$ & $85.87 \pm 18.12$ & $0.0000^{* * *}$ \\
iHOT12 score & $69.67 \pm 12.65$ & $96.57 \pm 17.56$ & $0.0000^{* * *}$ \\
\hline
\end{tabular}

Data were expressed as mean \pm SD (range) unless otherwise indicated $S D$ standard deviation

${ }^{*} P<0.05$; ${ }^{* *} P<0.01$; ${ }^{* *} P<0.001$

${ }^{\ddagger}$ Unpaired $t$ test. $P<0.05$ was defined as significant

symptom, and patients were satisfied with the outcome with no further consultation so far. No other common complications such as neurovascular injury, deep vein thrombosis, femoral head necrosis, and infection took place.

\section{Pre-operative radiographic findings}

Most cases (20 cases) presented with osteoarthritis of the affected hip in plain radiograph pre-operatively (Table 2), and all showed improvement in the postoperative plain radiograph at the final follow-up except for one case. Loose bodies could be identified in most cases (18 cases) on plain radiograph. The most typical presentation manifested as cloudy shadow around hip joint + with distinct ossified borders of each individual intra-articular radiopaque loose bodies (Fig. 2). Additional CT was needed in a rare condition (Fig. 2). Three cases of atypical radiological appearance of loose bodies with one large and unified fragment with ossified border detached from the acetabulum were identified (Fig. 5a). The fragment shares the same concavity with the acetabulum, making it easy to be dismissed. CT and MRI performance were all in consistency with radiograph appearance (Fig. 5b, c). Two cases with negative plain graphs were confirmed by MRI. Cam- and Pincer-type FAI were identified on the plain radiograph with bony hyperplasia at the junction of femoral head and neck

Table 2 Pre-operative radiographic findings

\begin{tabular}{llll}
\hline & & \multicolumn{2}{l}{ No. of hips (\%) } \\
\cline { 3 - 4 } & & Plain radiograph & MRI \\
\hline Osteoarthritis & Stage 0 & $1(4.8)$ & $1(4.8)$ \\
& Stage 1 & $11(52.4)$ & $11(52.4)$ \\
& Stage 2 & $7(33.3)$ & $7(33.3)$ \\
& Stage 3 & $2(9.5)$ & $2(9.5)$ \\
Lose bodies & Stage 4 & 0 & 0 \\
FAl & & $18(85.7)$ & $20(95.2)$ \\
& Cam & $5(23.8)$ & $5(23.8)$ \\
& Pincer & $1(4.8)$ & $1(4.8)$ \\
Synovial thickening & Mixed & $14(66.7)$ & $14(66.7)$ \\
\hline
\end{tabular}

and overcoverage of the acetabulum. All cases presented with synovial thickening and joint effusion on preoperative MRI. Labrum abnormality was presented in all cases, with 18 tear and 3 degeneration, detected on MRI. One patient with unspecific symptoms demonstrated both negative plain graph and MRI until non-ossified loose bodies were seen under arthroscopy. MRI was not performed as a routine postoperatively. At the final follow-up, most cases were absent of loose bodies (Fig. 2) except for two.

\section{Arthroscopic findings}

More cases showed acetabular abnormalities than the femoral head. Combined lesions at both ends could be seen in 19 patients. The cartilage of the femoral head was relatively well preserved compared to the acetabular cartilage, with more cases in grade I or II (15 cases) than grade III or IV (4 cases), which was the other way around at the acetabulum (7 cases in grade I or II and 13 cases in grade III or IV). Loose bodies could be seen under arthroscopy in all cases, with a mean number of 89 (ranging 7 520), the diameter of which ranging from 1 to $40 \mathrm{~mm}$ (Fig. 3). The morphology of loose bodies varied, from small chips scattered in the articular joint to large clustered aggregation wedged into the acetabulum fossa. We classified all the loose bodies we found according to their arthroscopic appearance on the basis of Milgram's theory of classification of loose bodies [3] (Fig. 4). In addition to this, we classified the type of large wedged clumps of loose bodies aggregated by multiple small ones separately as a single category (Fig. 4d). This category did not include randomly floating clumps of loose bodies. It referred specifically to the big clumps forged deeply into the acetabulum fossa. The diameter of these loose bodies was relatively bigger, which was the result of aggregation and fusion of piles of small ones. Some of these may broke into small pieces if clamped too hard. Probe was used to dig these loose bodies out of the fossa. We referred this type of loose bodies as the wedged clump type. Concurrent smaller loose bodies could be found in the central and peripheral compartment. All cases showed hypertrophy and hyperemia of the synovium (Table 3). Synovectomy was performed in all cases simultaneously. Labral abnormalities were consistent with MRI findings. Osteophytosis at the junction of head and neck and at the edge of acetabulum could be seen accordant with preoperative plain graph and was addressed accordingly.

\section{Discussion}

In this study, we performed a retrospective analysis of 21 patients with hip SC who underwent arthroscopy surgery. All but 1 patient have thus far reported a good outcome, with a pain-free joint, functional range of motion, 


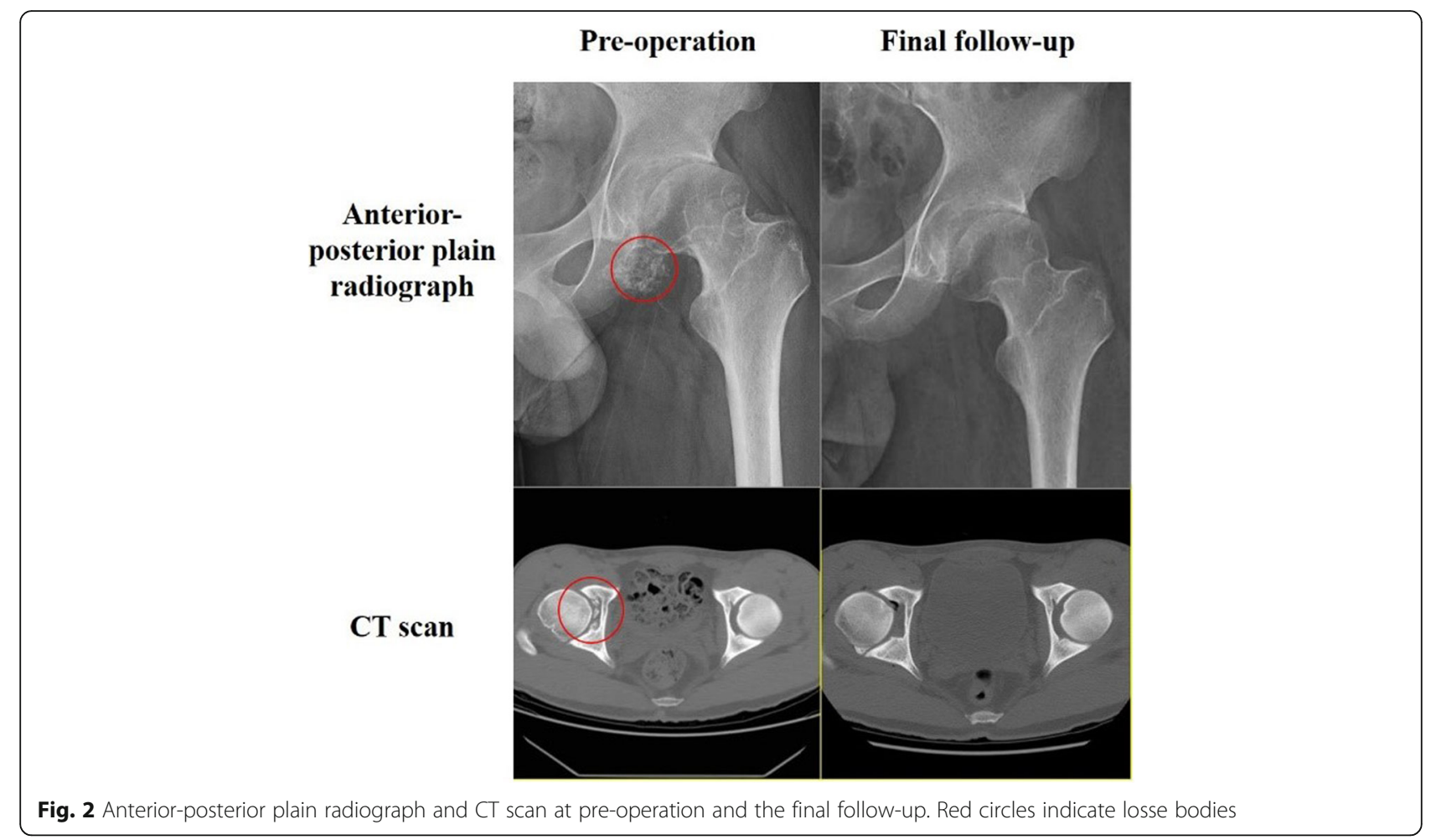

and return to their normal activities of daily living (including sport). Upon review of these cases, certain phenomenon deserved extra attention to be paid to.

Milgram classified loose bodies of SC into three types [3]: type I or early stage, where there are synovial-based masses without intra-articular loose bodies; type II or intermediate stage, where there are synovial-based masses and free intra-articular bodies; and type III or late stage, with multiple free osteochondral loose bodies without demonstrable synovial disease. In our study, six additional wedged clump type were found besides three types described by Milgram (Table 4). They appeared to be aggregation of loose bodies wedged, compressed, and accumulated at the horseshoe fossa, shaping like pomegranate seeds, which makes it difficult to be observed (Fig. 4d). There was no entanglement between the loose bodies and synovium, distinguishing them from Milgram type II or intermediate stage. The authors believe that

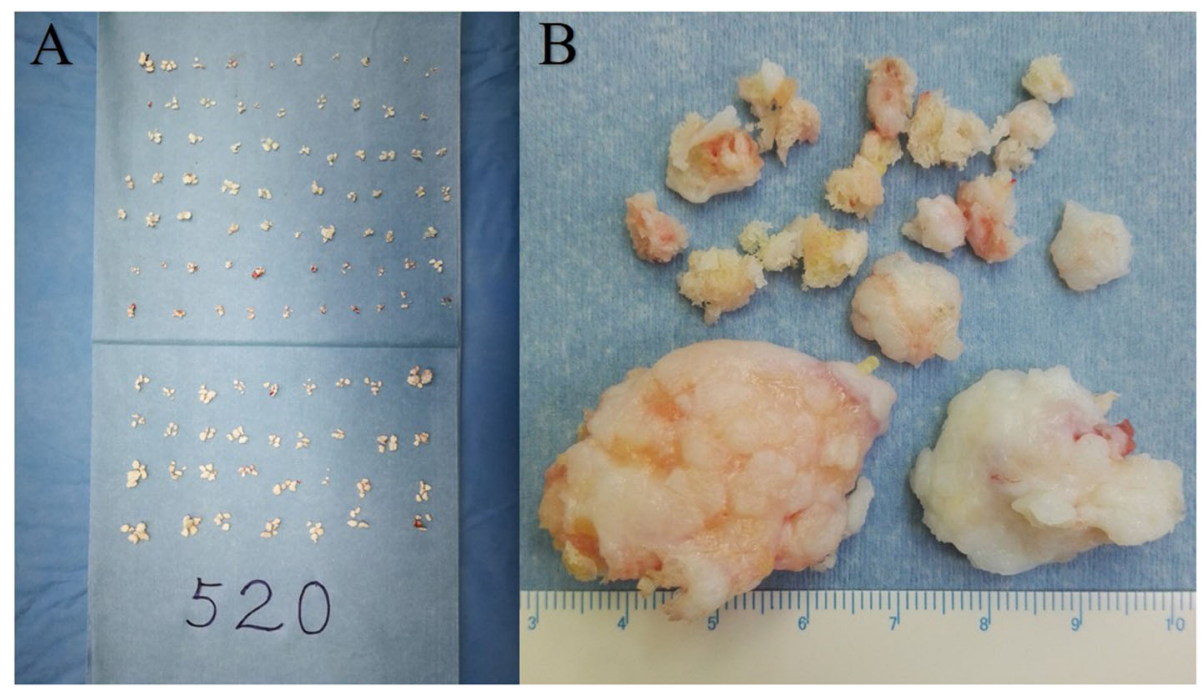

Fig. 3 Varies amounts and sizes of loose bodies. a: largest amount. b: largest size 


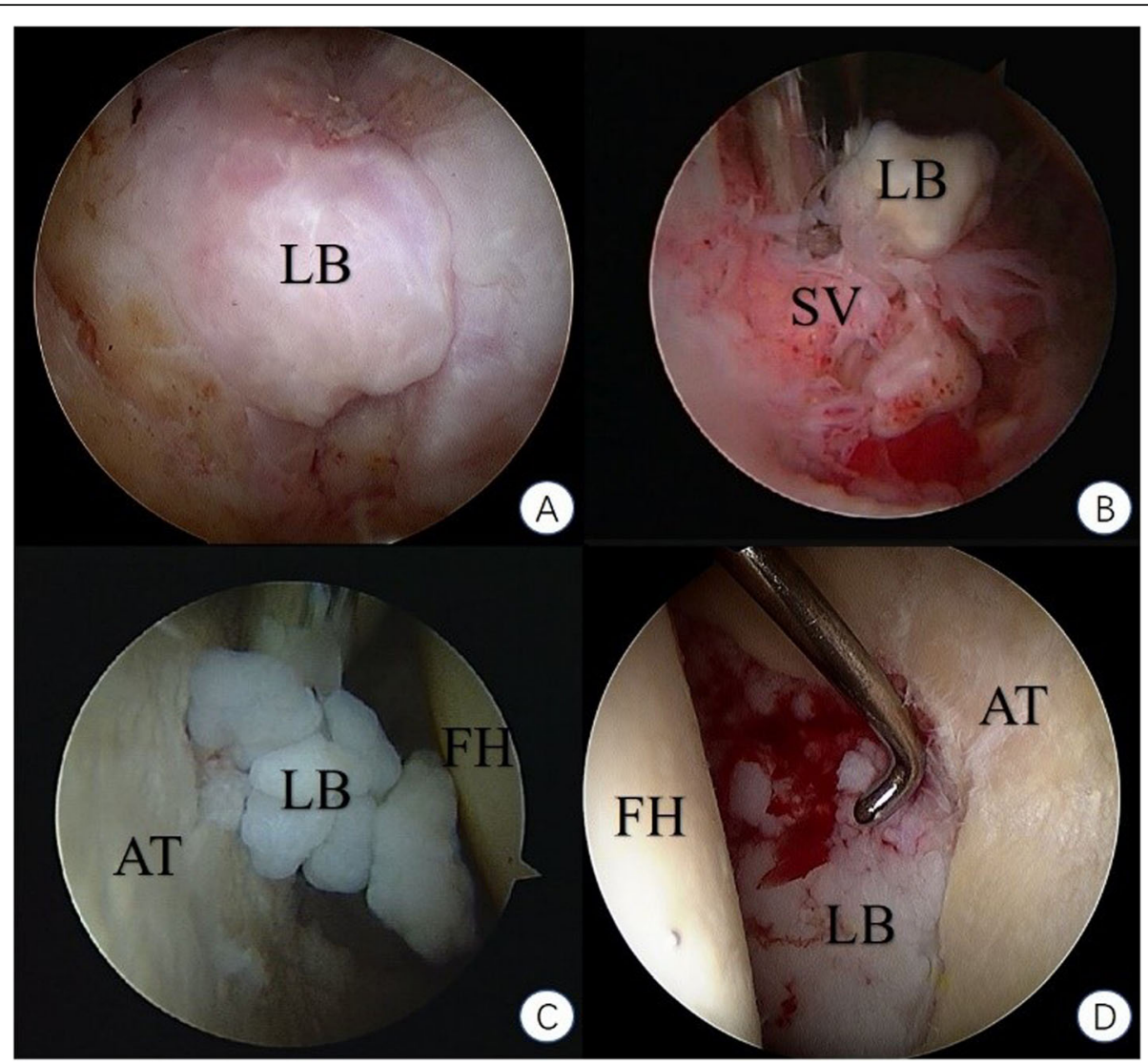

Fig. 4 Classification of all the loose bodies according to their arthroscopic appearance on the basis of Milgram's theory of classification of loose bodies. a: Milgram stage I. b: Milgram stage II. c: Milgram stage III. d: wedged-clump type. (LB: losse bodies. SV: synovium. FH: femoral head. AT: acetabulum.)

Table 3 Arthroscopic findings

\begin{tabular}{lll}
\hline & & $\begin{array}{l}\text { No. of hips } \\
(\%)\end{array}$ \\
\hline Synovial hypertrophy & & $21(100)$ \\
Labrum & Tear & $18(85.7)$ \\
& Fraying and degeneration & $3(14.3)$ \\
Osteophytosis & At junction of head and & $5(71.4)$ \\
& neck & \\
& At acetabulum & $1(4.8)$ \\
& At 2 ends & $14(66.7)$ \\
Acetabulum cartilage defect & Outerbridge stage 0 & $1(4.8)$ \\
& Outerbridge stage I & $3(14.3)$ \\
& Outerbridge stage II & $4(19)$ \\
& Outerbridge stage III & $6(28.6)$ \\
& Outerbridge stage IV & $7(33.3)$ \\
Femoral head cartilage defect & Outerbridge stage 0 & $2(9.5)$ \\
& Outerbridge stage I & $8(38.1)$ \\
& Outerbridge stage II & $7(33.3)$ \\
& Outerbridge stage III & $3(14.3)$ \\
& Outerbridge stage IV & $1(4.8)$ \\
\hline
\end{tabular}

the unique morphology of wedged clump-type loose bodies were due to the specific enarthrodial anatomy and biomechanical structure. Constant compression from the femoral head wedged numerous small loose bodies into the acetabulum fossa and forged these into a whole big clump. Therefore, as a classification designed for SC of all joints, Milgram's theory does not classify these as a separate category. Many physicians have described the large clumps of loose bodies formed by smaller ones and lodged into the acetabulum fossa [7, 14-17], proving its universal existence. We believe that this type of loose bodies distinguish themselves in terms of radiographic appearance, arthroscopic morphology, and surgical strategy to deal with. Therefore, we conclude our findings and hope it will provide certain guidance for fellow physicians.

SC's tendency of recurrence and 5\% of malignant transformation to synovial chondrosarcoma or chondrosarcoma make it cannot be neglected [18-20]. Due to the non-specific symptoms, the diagnosis of hip SC largely depends on radiographic findings. However, plain radiographs show the presence of loose bodies in only $50 \%$ of the cases [21], as 1/5 1/3 of loose bodies were not ossified 
Table 4 Types of loose bodies

\begin{tabular}{lllll}
\hline & Milgram stage I & Milgram stage II & Milgram stage III & Wedged clump type \\
\hline Male & $2(9.5)$ & $1(4.8)$ & $4(19)$ & $4(19)$ \\
Female & $1(4.8)$ & $2(9.5)$ & $5(23.8)$ & $2(9.5)$ \\
Total & $3(14.3)$ & $3(14.3)$ & $9(42.9)$ & $6(28.6)$ \\
\hline
\end{tabular}

$[22,23]$, making it notoriously elusive in imaging studies. In our study, loose bodies were detected in most cases on plain radiograph, with only three cases of negative finding. We conclude the relatively high resolution to the late stage development of most cases, which is corresponded with the arthroscopic findings. Most patients were reluctant to undergo any surgical management without actual evidence of pathological change until the symptoms became intolerable or radiopaque intra-articular loose bodies became ossified and visible on radiograph, which resulted in irreversible damage to the joint and consequently unsatisfying prognosis. MRI has become international gold standard in imaging $\mathrm{SC}$ in the pre-mineralization phase. Being a synovial pathology, morphologic changes are demonstrated best by contrast-enhanced T1-weighted fatsuppressed, axial T2-weighted, and coronal T1-weighted sequences [5]. As for the smaller loose body, MRI only presented as hip hydrops [9]. An atypical radiological appearance was identified on plain radiograph of three patients with wedged clump type loose bodies (Fig. 5). It was very similar to the image appearance of OCD (osteochondritis dissecans) with a large piece of ossified fragment dislodged from the acetabulum and fell into the joint space. Both the shape of wedged clump-type loose bodies and OCD fragment were found to be fitting the contour of the acetabulum, making them indistinguishable radiographically. Differential diagnoses between these two conditions were ambiguous, as neither symptoms nor radiological manifestation were specific. Arthroscopic surgery could serve as both treatment and diagnostic tool in this dilemma. In our study, three cases of the six wedged clump type presented with large ossified fragment fitting the concavity of acetabulum. Small amount of independent loose bodies were found in the central and peripheral

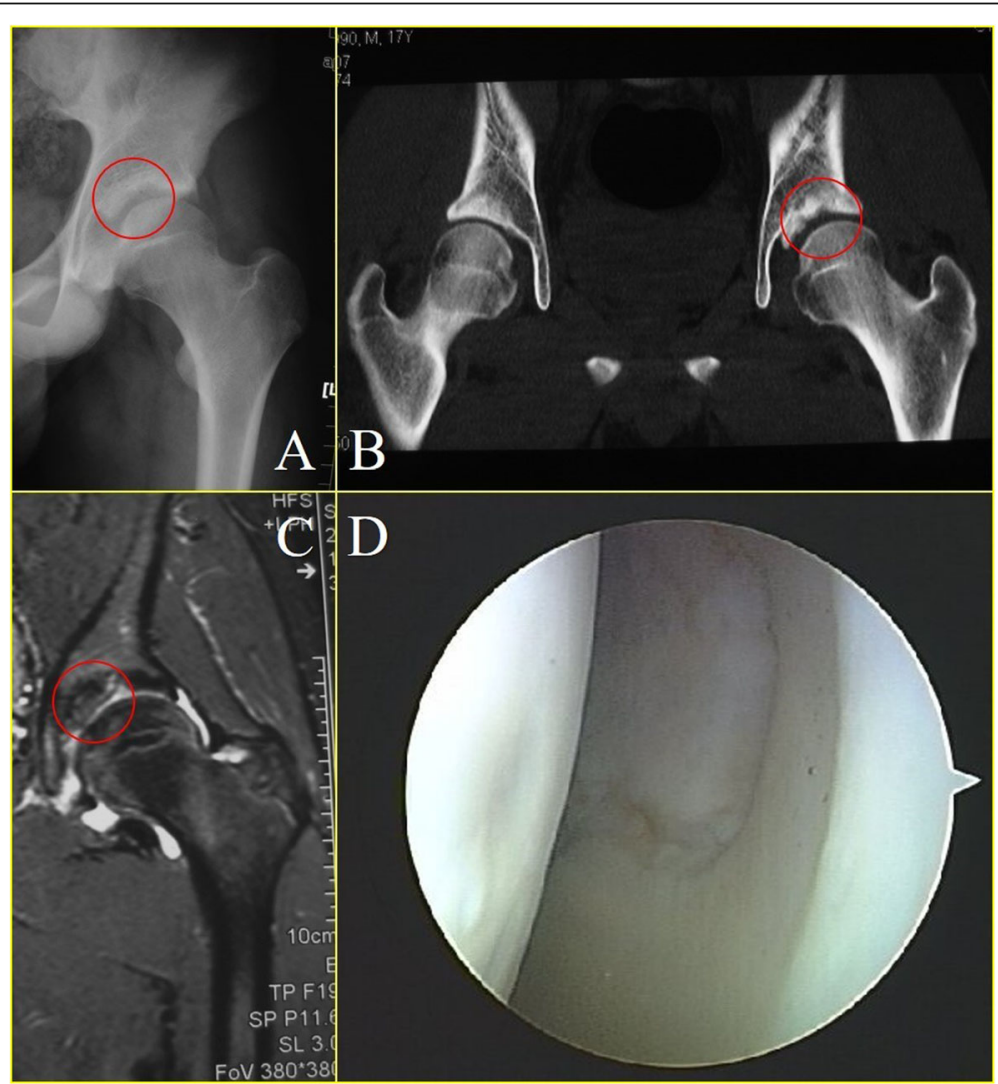

Fig. 5 An atypical radiological appearance identified on plain radiograph of three patients with wedged clump type loose bodies. Red circle indicate loose bodies. (a: anteroposterior plain radiograph. b: CT scan. c: MRI. d: arthroscopic view.) 
compartment under arthroscopic inspection. We believe that the relatively smaller quantity of scattered loose bodies was due to the fact that most loose bodies were compressed into the acetabular fossa and forged into one big clump. These additional small ossified loose bodies could be an indication for the diagnosis of SC, but were sometimes invisible on plain radiograph due to their insidious location or non-ossified status.

Different strategies were applied according to different types of loose bodies. Type I (Fig. 4a) was characterized by loose bodies confined in the synovium. At this stage, synovium remains its chondromateplastic potential, so the authors believe that a total synovectomy is essential to avoid recurrence and achieve a better prognosis. Loose bodies should be carefully searched and clamped out before synovectomy. Extra caution needs to be taken as to not break free and lost track of the loose bodies to reside intra-articularly when performing synovectomy. In type II (Fig. 4b), some "loose bodies" were not loosen any more. They were located at and attached to the free side of the synovium, making them generally hard to miss. Loose bodies should be taken care of at first, and then other pathological conditions. In our opinion, synovium should also be completely resected at this stage to prevent postoperative recurrence. In type III (Fig. 4c), scattered loose bodies were not confined in a specific area and moving freely in the joint capsule. The shape of loose body varies from round, oval, rice-shaped to antlers, and so was the size. Flexing, extending, and rotating of the hip joint after relaxation and traction during the operation can effectively avoid the missing of loose bodies. Observation of the lateral part of the hip was more accessible when the hip was fully flexed and external rotated, which is relatively easy compared to the medial part, which could be best inspected with the hip extended and internal rotated. The following methods were taken to avoid blind angle: (1) interchange the approach of arthroscopy and surgical instrument, (2) using arthroscopy of $30^{\circ}$ and $70^{\circ}$ in turn, and (3) expanding the internal access of the approach to increase the range of the movement of scope. Synovectomy should be restricted to the hypertrophy and hyperemia area. When dealing with the wedged clump type (Fig. $4 \mathrm{~d}$ ), due to the embedding of the loose bodies into the acetabular fossa, it is difficult for the general surgical instruments to reach to. Curved scraper and slide winder blade were needed to remove all the loose bodies out. Some of the wedged big ones were in fact the aggregation of many small loose bodies hinged together, each with intact calcified or cartilaginous surface of its own. Clamping too hard may result in morselization of the aggregation and scatter of the floating loose bodies, increasing the complexity and time duration of the surgery. However, when the wedged loose bodies were too big to maneuver, breaking down of the aggregation might be inevitable. A half pipe cannula might be used to secure the easy passage of the small chipped loose bodies.

The precise congruency of the articulation of acetabulum and femoral head makes the hip an intricate structure. Despite its relatively large size, the intra-articular space of the hip joint for surgical maneuverability is relatively insufficient. T-shaped capsulotomy was first brought up by Horisberger et al. [24] for the management of FAI. It is an incision between the ALA, AMA, and DALA on the capsule along the axis of femoral neck, along the iliofemoral ligament, and perpendicular to the intertrochanteric line (Fig. 6). It aims to increase the exposure for better arthroscopic surveillance of peripheral compartment and space for instrument operability to deal with certain central compartment pathology. In this study, it was carried out in each case to facilitate instrument maneuverability and thorough inspection of capsular recess. It allows the removal of loose bodies as many as possible and does not add substantially to the case time, complications, or complexity. Moreover, a complete exposure becomes more accessible with this cut and therefore result in a more thorough synovectomy without taking the risk of a series of potential complications resulted by dislocation of the hip joint. In addition to that, FAI is known to be commonly

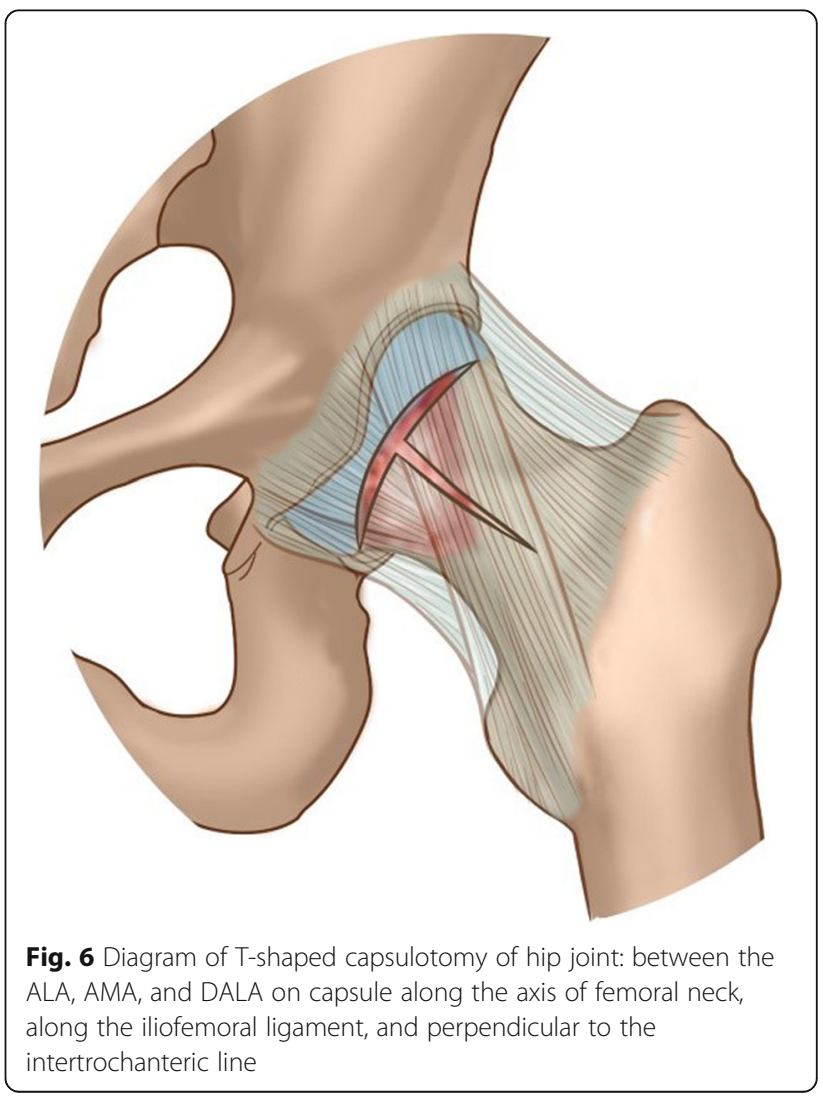


associated with hip SC [25, 26], along with labrum abrasion and tear. T-shaped capsulotomy facilitates not only the resection of osteophytes at the junction between the femoral head and neck, but also debridement, stitches, and suture anchor placement to manage the labral pathology.

It should be noted that the following problems still exist in this study: limited number of cases were included in this study, and with a larger size of samples, the credibility will be more authoritative. Longer follow-up time is also needed for better credibility. We believe that our experience and operative guidance followed could help to achieve a more satisfactory outcome and to lower the rate of recurrence and other complications. Hopefully, this theory could provide theoretical guidance and support for clinicians to perform better diagnosis and treatment for patients.

\section{Conclusion}

Arthroscopic management with T-shaped capsulotomy of hip SC brought an excellent postoperative outcome with no recurrence, shorter hospitalization and rehabilitation time, and faster return to life. Wedged clump loose bodies manifested distinguishably in terms of radiological appearance, arthroscopic morphology, and surgical strategy.

\section{Code availability}

Not applicable.

\section{Declarations}

We declare that we do not have any commercial or associative interest that represents a conflict of interest in connection with the work submitted.

\section{Authors' contributions}

All authors contributed to the study conception and design. Material preparation, data collection, and analysis were performed by Yufeng Liu, Ji Li, Ning Ma, Mingyang An, Feng Gao, Bo Hu, Ming Lu, and Weiqi. The first draft of the manuscript was written by Yufeng Liu, and all authors commented on previous versions of the manuscript. All authors read and approved the final manuscript. Conceptualization, editing, and supervision: Chunbao Li, Zhigang Wang, and Yujie Liu.

\section{Funding}

This research received grant from the 2017 Beijing Nova program [XX2018038], Medical Science Program for Top Young Scholar [19QNP070], and General Program of General Logistics Department [CWS14J067].

\section{Availability of data and materials}

All data generated or analyzed during this study are included in the article.

\section{Ethics approval and consent to participate}

Ethical approval was waived by the local Ethics Committee of PLA General Hospital in view of the retrospective nature of the study and all the procedures being performed were part of the routine care.

\section{Consent for publication}

Informed consent was obtained from each patient on the day of admission.

\section{Competing interests}

The authors declare that they have no conflict of interest.

\section{Author details}

'Department of Sports Medicine, Chinese PLA General Hospital, Beijing 100853, China. ${ }^{2}$ Institute of Orthopedic, Chinese PLA General Hospital, Beijing 100853, China. ${ }^{3}$ Department of Sports Injury and Arthroscopy Surgery, National Institute of Sports Medicine, Beijing 100061, China. ${ }^{4} T$ he Second Department of Orthopedics, Beijing Chaoyang Integrative Medicine Emergency Medical Center, Beijing 100023, China.

Received: 2 April 2020 Accepted: 25 August 2020

Published online: 11 September 2020

\section{References}

1. Bai L, Wang $H$, Zhang $Y$, et al. Synovial chondromatosis: clinical analysis of 13 cases. Zhonghua wai ke za zhi [Chinese journal of surgery]. 1999; 37:364-5.

2. Ackerman D, Lett $P$, Galat DD Jr, et al. Results of total hip and total knee arthroplasties in patients with synovial chondromatosis. The Journal of arthroplasty. 2008;23:395-400.

3. Milgram JW. Synovial osteochondromatosis: a histopathological study of thirty cases. J Bone Joint Surg Am. 1977;59:792-801.

4. Neumann JA, Garrigues GE, Brigman BE, et al. Synovial chondromatosis. JBJS Rev. 2016:4:01874474-201605000-201600005.

5. Murphey MDVJ, Fanburg-Smith JC, Gajewski DA. Imaging of synovial chondromatosis with radiologic-pathologic correlation. Radiographics. 2007: 27(5):1465-88

6. Duif $C$, von Schulze PC, Ali A, et al. Primary synovial chondromatosis of the hip - is arthroscopy sufficient? A review of the literature and a case report. Technol Health Care. 2014;22:667-75.

7. Zini R, Longo UG, de Benedetto $M$, et al. Arthroscopic management of primary synovial chondromatosis of the hip. Arthroscopy. 2013;29: 420-6.

8. Monu JOM. Synovial osteochondromatosis. eMedicine. 2004:1-13.

9. Lim SJ, Department of Orthopedic Surgery SUSOMSMCK-KSSKPYS. Operative treatment of primary synovial osteochondromatosis of the hip. Surgical technique. J Bone Joint Surg Am. 2007:89(Suppl 2):232

10. Schoeniger R, Naudie DD, Siebenrock KA, et al. Modified complete synovectomy prevents recurrence in synovial chondromatosis of the hip. Clin Orthopaedics Related Res. 2006;451:195-200.

11. Lin RC, Lue KH, Lin $\mathrm{Zl}$, et al. Primary synovial chondromatosis mimicking medial meniscal tear in a young man. Arthroscopy. 2006;22: 803.e801-3.

12. Hermann EAF, Klein M, Kenan S, Lewis M. Synovial chondromatosis. Skeletal Radiol. 1995;24:298-300.

13. de Sa D, Horner NS, MacDonald A, et al. Arthroscopic surgery for synovial chondromatosis of the hip: a systematic review of rates and predisposing factors for recurrence. Arthroscopy. 2014;30(1499-1504): e1492.

14. Boyer $\mathrm{T}$, Dorfmann $\mathrm{H}$. Arthroscopy in primary synovial chondromatosis of the hip: description and outcome of treatment. J Bone Joint Surg Br. 2008; 90:314-8.

15. Ferro FP, Philippon MJ. Arthroscopy provides symptom relief and good functional outcomes in patients with hip synovial chondromatosis. J Hip Preserv Surg. 2015:2:265-71.

16. Lee J-B, Kang C, Lee C-H, et al. Arthroscopic treatment of synovial chondromatosis of the hip. Am J Sports Med. 2012;40:1412-8.

17. Marchie A, Glassner PJ, Panuncialman I, et al. Technical pearls for hip arthroscopy in the management of synovial chondromatosis. Am J Orthopedics (Belle Mead, NJ). 2012;41:284-7.

18. Blumlein HPP, Schneider $\mathrm{H}$, Wunderlich $\mathrm{T}$. Benign and malignant chondromatosis of the joints. A contribution to the clinical aspects and histology of the disease. Z Orthoplhre Grenzgeb. 1980;118:8-14.

19. Blokx WA, Rasing LA, Veth RP, et al. Late malignant transformation of biopsy proven benign synovial chondromatosis: an unexpected pitfall. Histopathology. 2000;36:564-6.

20. Davis RI, Hamilton A, Biggart JD. Primary synovial chondromatosis: a clinicopathologic review and assessment of malignant potential. Hum Pathol. 1998;29:683-8.

21. Christensen JHPJ. Synovial chondromatosis. Acta Orthop Scand. 1975;46: 919-25.

22. Helms CAMN, Anderson MW, Kaplan PK, Dussault R. Arthritis and cartilage Musculoskeletal MRI. 2nd ed; 2009. p. 116-7. 
23. Kim SH, Hong SJ, Park JS, et al. Idiopathic synovial osteochondromatosis of the hip: radiographic and MR appearances in 15 patients. Korean J Radiol. 2002;3:254-9.

24. Horisberger M, Brunner A, Herzog RF. Arthroscopic treatment of

femoroacetabular impingement of the hip: a new technique to access the joint. Clin Orthop Relat Res. 2010;468:182-90.

25. Abolghasemian M, Gharanizadeh K, Kuzyk P, et al. Hips with synovial chondromatosis may display the features of femoroacetabular impingement. J Bone Joint Surg Am. 2014;96:e11.

26. Fukui K, Kaneuji A, Amaya S, et al. Synovial osteochondromatosis of the hip with femoroacetabular impingement and osteoarthritis: a case report. J Orthop Surg (Hong Kong). 2013;21:117-21.

\section{Publisher's Note}

Springer Nature remains neutral with regard to jurisdictional claims in published maps and institutional affiliations.

Ready to submit your research? Choose BMC and benefit from:

- fast, convenient online submission

- thorough peer review by experienced researchers in your field

- rapid publication on acceptance

- support for research data, including large and complex data types

- gold Open Access which fosters wider collaboration and increased citations

- maximum visibility for your research: over $100 \mathrm{M}$ website views per year

At BMC, research is always in progress.

Learn more biomedcentral.com/submissions 